La physique de Laplace était essentiellement moléculaire.

\section{Grâce à Maxwell, Boltzmann}

et Van der Waals, cette physique devint la base même de la

thermodynamique moléculaire statistique. Ce fut Jean Perrin

qui proposa, en 1908, une

preuve expérimentale des plus élégantes du bien-fondé de cette théorie.

Jean Perrin a aussi joué un rôle majeur dans la politique scientifique de la France des années 1930 : il est à l'origine de la création du CNRS et a fondé le Palais de la découverte à Paris.

\title{
Éloge de Jean Perrin (1870-1942)
}

Henk Kubbinga (h.kubbinga@home.nl)

Université de Groningen, Bilderdijklaan 109, NL-9721-PT, Groningen (Pays-Bas)

Membre du groupe d'histoire de la physique de la Société européenne de Physique (EPS)

Jean [Baptiste] Perrin entre en 1891 à l'École normale supérieure de Paris. Le jeune normalien avait fait un parcours typiquement français : école primaire et collège en province, lycée à la capitale. L'École était fameuse pour son gauchisme, mais ses élèves n'en portaient pas moins des uniformes et s'enorgueillissaient de leurs traditions, tout en méprisant leurs collègues de la Sorbonne. Les mathématiques faisaient la différence ; Perrin n'y avait aucun problème. Il choisit Marcel Brillouin (18541948) comme directeur d'études, le même qui avait introduit, en France, les célèbres Vorlesungen über Gastheorie de Ludwig Boltzmann.

Tout en préparant son doctorat d'État ès sciences, Perrin débute en 1895 sur la scène internationale, en expérimentateur extrêmement doué, avec la publication d'une démonstration convaincante du fait que la charge des (particules des) rayons cathodiques est négative (fig. 1) [1]. En combinaison avec d'autres travaux sur les rayons de Röntgen, il obtient, en 1896, le Prix James P. Joule de la Société royale de Londres. Quel essor pour un jeune homme n'ayant guère que vingt-six ans !

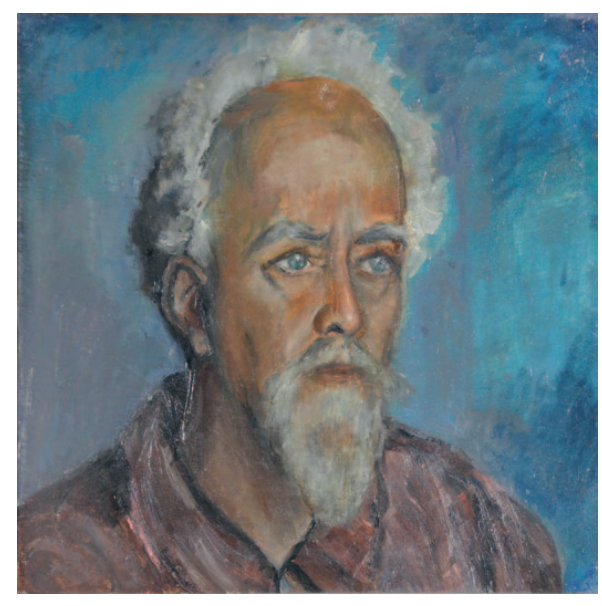

Jean Perrin, tableau réalisé par sa fille Aline Lapique-Perrin (vers 1926 ; huile sur toile, 34 × 37 cm, collection de la famille Perrin).

\section{Un aller-retour : de la physico-chimie à la physique}

À l'ENS, Perrin est chargé d'un nouvel enseignement : un cours de physico-chimie, science inaugurée très récemment par Van't Hoff, Ostwald et Arrhenius. Ceci l'amène à composer une monographie qui fera date : le Traité de chimie physique. Les principes (1903). Parmi ses amis, il y a Pierre Curie et Marie Curie-Sklodowska, ses voisins du boulevard Kellermann, dans le XIII ${ }^{\mathrm{e}}$ arrondissement de Paris, et Paul Langevin, ancien élève de Pierre Curie.

$\gg>$
Cet article est essentiellement une traduction par l'auteur de l'original, en langue anglaise, paru dans Europhysics News, 44, n5 (2013), pp. 16-18.

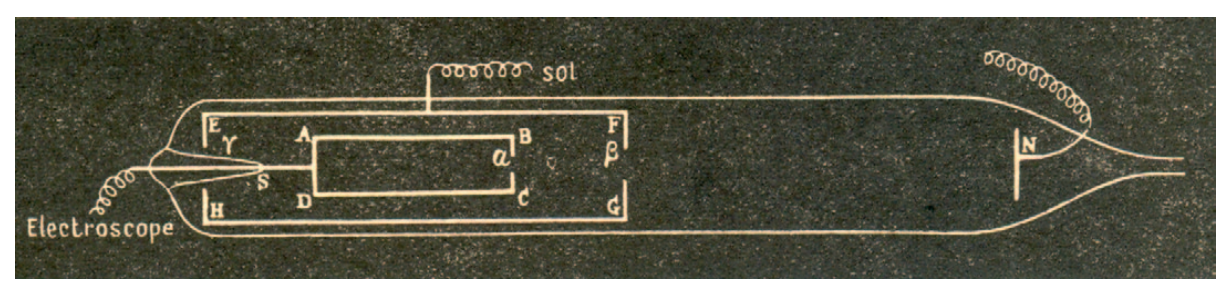

1. Démonstration de la charge négative des particules responsables des phénomènes dans les tubes à décharge. $N$ représente la cathode ; le cylindre EFGH est l'anode (mise à la terre). À travers les ouvertures $\beta$ et $\alpha$, le pinceau non perturbé de rayons cathodiques atteint la cage de Faraday $A B C D$, qui a été branchée sur un électroscope à feuille d'or. À l'aide d'un électroaimant, ce pinceau peut être dévié, si bien qu'il n'entre plus dans l'ouverture $\beta$ et n'atteint plus ABCD [1]. 
Ces gens menaient une vie sobre, tout comme les Perrin, Jean et sa femme Henriette. Ils se rencontraient régulièrement, le plus souvent côté Perrin, où le maître d'hôtel jouait du piano. La nature de l'atome figurait à l'ordre du jour, certes, et donnait lieu à des débats des plus vifs. Perrin jouait avec un modèle s'inspirant du système solaire - un «soleil » chargé positivement, entouré de " planètes » négatives -, mais n'insistait pas.

Un moment capital fut l'invention, en 1903, d'une nouvelle technique microscopique par l'Allemand Henry Siedentopf et l'Autrichien Richard Zsigmondy. Du jour au lendemain, une ancienne énigme refit surface dans le subconscient scientifique : le mouvement brownien. Ce phénomène avait été découvert, à la fin des années 1820, par l'Anglais Robert Brown. Ce dernier avait remarqué que toutes sortes de granules infimes - des grains de pollen, entre autres -, en suspension dans des liquides, se mouvaient sans cesse en décrivant des trajectoires en zigzag des plus irrégulières. En 1903, ce sont les particules dites colloïdales qui paraissaient manifester ce mouvement, comme Siedentopf et Zsigmondy le constatèrent avec leur ultramicroscope, dont l'illumination latérale permettait d'observer ces particules sur un fond obscur. Le mouvement irrégulier de ces dernières faisait penser, irrésistiblement, à celui postulé dans la théorie des gaz pour les molécules et à leur libre parcours entre deux chocs : plus les particules sont grandes et/ou la température basse, plus le mouvement est lent. Perrin, de son côté, avait été harcelé par les philosophes de la Sorbonne qui s'interrogeaient sur la validité ou non, de l'utilisation du modèle moléculaire en sciences physiques et naturelles. Après tout, il s'agit de petites entités invisibles, qui se soustraient même au nouveau microscope. La réponse de Perrin était époustouflante dans sa simplicité [2]. C'est qu'il renvoyait aux microbes de ses contemporains Louis Pasteur et Robert Koch, en demandant aux philosophes de s'imaginer un état hypothétique de la science, à savoir, sans l'invention du microscope. Dans un tel état, Koch et Pasteur - disons l'ensemble des médecins - auraient quand même pu conclure que les maladies contagieuses sont produites par des êtres vivants infimes, capables d'infecter une victime et de s'y reproduire avant de s'attaquer à de nouvelles victimes. Des mesures prophylactiques parfaitement effectives auraient pu être développées dans cette hypothèse, en essayant des substances chimiques appropriées sur des matières infectées. Il n'empêche que le microscope était là et qu'il a énormément facilité la recherche. Concernant les molécules, toutefois, la situation est inverse : il n'y avait pas encore de microscope suffisamment puissant pour les rendre visibles, mais les scientifiques n'en avaient pas moins réussi à faire évoluer de manière cohérente la physique et la chimie à partir de l'idée même de molécules composées d'atomes. Aujourd'hui, au début du $\mathrm{XXI}^{\mathrm{e}}$ siècle, où nous vivons l'essor de la microscopie monomoléculaire sinon monoatomique, il est facile d'apprécier la brillante logique "perrinienne ".

\section{Le mouvement brownien}

L'invention de Siedentopf et Zsigmondy ouvrait des voies nouvelles à la recherche sur les particules colloïdales. Dans ce contexte, il n'est pas étonnant que le mouvement brownien se soit imposé petit à petit comme thème crucial, ne serait-ce que parce qu'il affectait directement le cœur même de la physique contemporaine, à savoir, la théorie cinétique des gaz.

En 1905-1906, Einstein et Smoluchowski publiaient leurs analyses. Einstein montrait que le parcours en zigzag des particules colloïdales pouvait être déduit de ce qu'il appelait « la théorie moléculaire-cinétique de la chaleur ». L'un de ses arguments dérivait de la pression osmotique, à laquelle on pouvait s'attendre dans le cas des suspensions : en effet, la seule différence entre des molécules dissoutes et des particules en suspension réside dans leur taille. En conséquence, si les molécules se déplacent au hasard dans toutes les directions, elles doivent, elles aussi, effectuer un tel mouvement, même si leur libre parcours est plus petit. Quelque chose de semblable s'applique aux phénomènes de diffusion. À l'évidence, la translation et la rotation de ce genre de particules devraient être analysées dans l'approche de la physique statistique de Maxwell-Boltzmann. Alors, en principe, la vitesse thermique de toute particule - imaginée à l'état gazeux, donc libre - peut être calculée à l'avance, à moins que l'on ne connaisse sa masse. Une fois mise en suspension dans un liquide plus ou moins visqueux, la friction tendrait naturellement à ralentir la particule. L'application de la loi de Stokes permet alors une évaluation quantitative. Le Polonais Marian
Smoluchowski, de son côté, raisonnait inversement : il soulignait l'importance des chocs entre les molécules du liquide et les particules en suspension. C'est dans ce contexte que J. Perrin se lance dans une recherche expérimentale des phénomènes accompagnant le mouvement brownien.

\section{Laplace et Perrin : granules et molécules}

Dans son chef-d'œuvre Traité de mécanique céleste, Laplace avait déduit une formule pour la décroissance observée de la pression $p$ de l'air avec l'altitude $h$. L'essentiel en était que cette décroissance était sujette à une loi logarithmique $: \ln \left(p_{0} / p\right)=\mathrm{C} \cdot h$. La constante $\mathrm{C}$ dans cette dernière formule avait été établie en comparant la pression de l'air au pied et au sommet d'une montagne d'une hauteur exactement connue, le célèbre

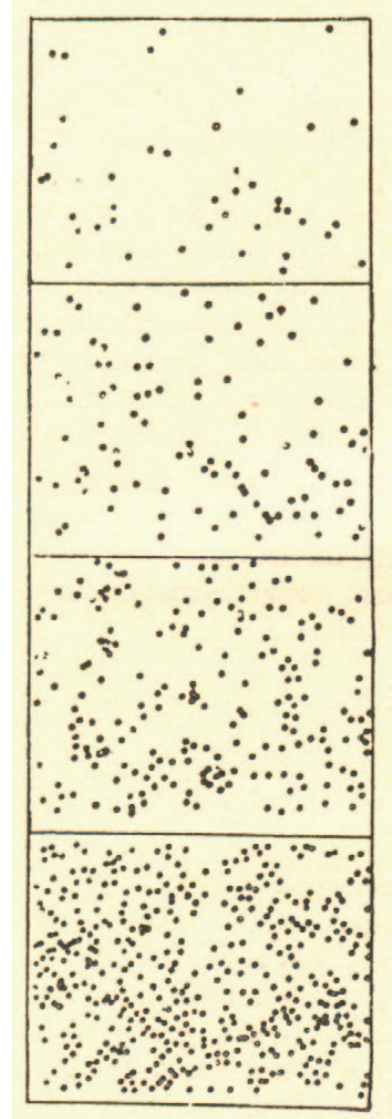

2. Répartition d'équilibre de grains de gommegutte de taille pratiquement uniforme, en suspension dans une solution liquide. Dessins faits d'après quatre photographies, prises à des hauteurs différant de $10 \mu \mathrm{m}$. Les grains s'accumulent dans le niveau inférieur. Le diamètre moyen des grains était de $0,6 \mu m$ (réf. [3], p. 57). 
Puy-de-Dôme, en Auvergne. Cela permet aux premiers aéronautes scientifiques, Gay-Lussac et Biot, de calculer la hauteur atteinte avec leur ballon, en 1804: 6977 mètres au-dessus de Paris.

Perrin avait remarqué que des suspensions de grains de gomme-gutte (une résine végétale jaune) en repos - disons, dans une éprouvette - subissaient une sédimentation, de telle manière qu'il s'y installait un gradient d'intensité de couleur : ceci lui fit penser à la loi logarithmique de Laplace [3]. Si, en effet, l'analogie entre granules et molécules était correcte, le nombre de granules devait diminuer selon la formule $\ln \left(n_{0} / n\right)=\mathrm{C} \cdot h$. Le nouvel ultramicroscope de Zsigmondy et Siedentopf lui permettait alors de tester cette formule. En effet, grâce à la petite profondeur focale de ce microscope, Perrin pouvait simplement prendre des photographies à différentes hauteurs et compter, sur celles-ci, le nombre de granules dans le champ de vision, pour établir sa variation en fonction de la hauteur. Or, ce nombre paraissait se diviser en deux pour chaque élévation d'un montant donné, par exemple tous les $10 \mu \mathrm{m}$ pour des grains de diamètre $0,6 \mu \mathrm{m}$ (fig. 2). Parfait ! C'était dire en même temps que l'analogie initiale était justifiée. Inversement : puisque les granules existent manifestement, les molécules, elles aussi, existent en vérité. À l'aide de ces granules, Perrin réussit même à déterminer le nombre de molécules dans une molécule-gramme, la mole de nos

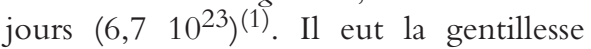
d'attribuer à ce nombre le nom du spécialiste italien de l'état gazeux, Amedeo Avogadro (1776-1856).

\section{Nouvelle perspective : rien que de la physique}

Dans l'œuvre de Jean Perrin, il y a une filiation directe avec la physique de Laplace : les deux sont essentiellement moléculaires. Il n'empêche que c'est Perrin qui a accompli ce que son illustre prédécesseur avait négligé, à savoir la détermination des dimensions moléculaires. Si les philosophes, eux, se croyaient légitimés à interférer, c'était en vain. La physique ne leur doit rien : elle est autonome et produit sa propre philosophie. L'exploit brillant de Perrin lui valut le Prix Nobel de physique en 1926.

Jean Perrin fut un excellent pédagogue. Il expose les raisonnements et les expériences

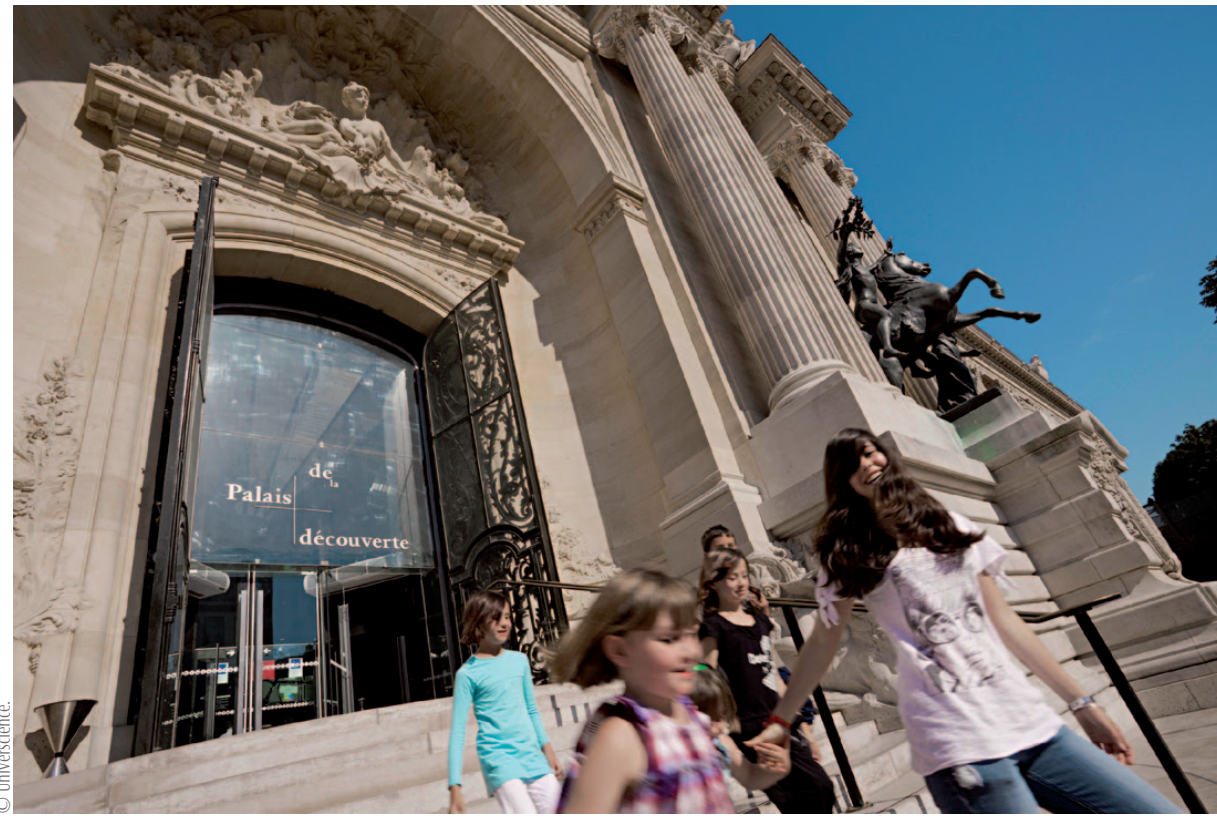

Le Palais de la découverte (Paris, 8 e arrondissement), fondé en 1937 par Jean Perrin.

qui ont abouti à la théorie atomique dans un remarquable petit livre, tout à fait lisible par un large public et dont l'introduction est restée célèbre jusqu'à aujourd'hui : Les atomes, publié en 1913. Ce best-seller, traduit en plusieurs langues, a été réédité récemment en France [4].

\section{Science et politique dans les années 1930}

Dès 1930, soucieux de l'organisation de la recherche en France, Jean Perrin exposa à l'Académie des sciences un projet de création d'un Service national de la Recherche scientifique. Plus tard, Perrin et ses compagnons socialistes rejoindront le gouvernement français. En sa qualité de sous-secrétaire d'État à la recherche scientifique (1936-1938), il participera activement à la création (effective en octobre 1939) du Centre National de la Recherche Scientifique (CNRS), dans le but affiché de revigorer la vie académique dans les échelons supérieurs. Il fonde en 1937 le Palais de la découverte, afin de montrer à tous les publics " la science en train de se faire ». En 1941, en pleine guerre mondiale, Perrin et sa famille se rendent aux ÉtatsUnis, où il décéde l'année suivante.

(1) Les expériences de Perrin ont montré que cette élévation, qui entraîne une raréfaction donnée des grains, varie en raison inverse de la masse du grain. L'extrapolation de cette loi à la variation de densité d'un gaz en fonction de l'altitude, permet d'obtenir le poids de la molécule de gaz, et donc le nombre d'Avogadro.
Remerciements

Je remercie M. Daniel Ricquier, Délégué aux Relations Internationales de l'Académie des sciences de l'Institut de France, qui m'a permis de consulter le fond Jean Perrin des archives de l'Académie. L'appui chaleureux de la famille Perrin - dans les personnes de Mme Françoise Chappuis-Perrin et de MM. Nils et David, ses petits-enfants - m'a été très cher. Les droits de reproduction de la photo du tableau sont les leurs.

\section{L'auteur}

Henk Kubbinga est un historien des sciences de l'Université de Groningen (Pays-Bas), membre du Groupe d'histoire de la physique de la Société européenne de Physique. Il a obtenu un doctorat de $3^{\mathrm{e}}$ cycle (1983) et une habilitation (1996) à I'EHESS de Paris. Ses thèses ont été résumées dans la monographie L'Histoire du concept de "molécule » (jusqu'à c. 1925) (en trois volumes ; Paris, Springer-Verlag France, 2001). Pour les détails de l'exploit de Perrin, voir le volume 3, pp.1131-1156.

\section{Références}

1- J. Perrin, Comptes rendus de l'Académie des sciences, 121 (1895) 1130

2• J. Perrin, Revue Scientifique, 15 (1901) 449

3• J. Perrin, « Mouvement brownien et réalité moléculaire ", Annales de Chimie et de Physique (8e série), 18 (1909) 5-114.

4- J. Perrin, Les atomes, Librairie Félix Alcan (1913). Réédité en 1991 par Flammarion, avec une préface de Pierre-Gilles de Gennes. 